\title{
Chinese Herbal Medicine (Zi Shen Qing) for Mild-to-Moderate Systematic Lupus Erythematosus: A Pilot Prospective, Single-Blinded, Randomized Controlled Study
}

\author{
Linda L. D. Zhong, ${ }^{1}$ Zhao Xiang Bian, ${ }^{1}$ Jun Hua Gu, ${ }^{2}$ Xin Zhou, ${ }^{3}$ Yu Tian, ${ }^{2}$ \\ Jian Chun Mao, ${ }^{2}$ and Xiang Jun Chen ${ }^{2}$ \\ ${ }^{1}$ Clinical Division, School of Chinese Medicine, Hong Kong Baptist University, Hong Kong \\ ${ }^{2}$ Department of Rheumatology, Longhua Hospital, Shanghai University of Traditional Chinese Medicine, \\ 725 Wan Ping South Road, Shanghai, China \\ ${ }^{3}$ Department of Pharmacy, Longhua Hospital, Shanghai University of Traditional Chinese Medicine, \\ 725 Wan Ping South Road, Shanghai, China
}

Correspondence should be addressed to Xiang Jun Chen; chenxiangjun0321@gmail.com

Received 9 February 2013; Revised 8 April 2013; Accepted 10 April 2013

Academic Editor: Aiping Lu

Copyright (C) 2013 Linda L. D. Zhong et al. This is an open access article distributed under the Creative Commons Attribution License, which permits unrestricted use, distribution, and reproduction in any medium, provided the original work is properly cited.

Objective. The aim of this study is to investigate the effectiveness and safety of a Chinese herbal formula Zi Shen Qing (ZSQ) in the treatment of systematic lupus erythematosus (SLE) in Chinese patients. Methods. A randomized controlled trial was conducted over 12 weeks in 84 Chinese patients who reported total scores of SLE Disease Activity Index-2000 (SLEDAI-2000) was from 5 to 14. The primary outcome was the changes of the SLEDAI-2000. The secondary outcomes included score changes of Chinese Medicine Syndromes (CMS), the changes of steroid dosage, the incidence of disease flare-up and biologic markers. Results. ZSQ significantly reduced SLEDAI-2000, the total scores of CMS in the treatment group compared with the controlled group $(P<0.05)$. Superiority of ZSQ over controlled group was also observed with greater improvement in the withdrawal dosage of corticosteroids and the incidence of disease flare-up $(P<0.05)$. There were no serious adverse events, and safety indices of whole blood counts, renal and liver functions were normal, both before and after the treatment. Conclusion. ZSQ is safe and effective for decreasing SLE disease activity and withdrawal dosage of corticosteroids in the mild to moderate SLE patients with "Deficiency of Qi and Yin" Pattern.

\section{Introduction}

Systematic lupus erythematosus (SLE) is a typical autoimmune disease which affects a variety of organ systems and markedly causes significant physical disfigurement, morbidity, and occasionally mortality [1-3]. Patients with SLE also develop distinct immunologic abnormalities, particularly in the antinuclear, anticytoplasmic, and antiphospholipid antibodies [4-6]. The overall population prevalence is 400 to 100 per 100,000 women, depending on age groups and demographic factors [7]. Although available therapies, such as corticosteroids, hydroxychloroquine, and the new immunosuppressive drugs, have improved the overall survival and severe organ damage, there remains a significant unmet need for quality of life as well as more safe and effective treatments [8-10]. With the unsatisfactory response to current conventional treatments [11], many patients seek help from traditional Chinese medicine (TCM), mostly by taking Chinese herbal medicine (CHM) [12].

According to TCM, SLE can be defined as rheumatism (Bi Zhen), edema, and palpitation of heart due to different organic damage and in general divided into excessive and deficient syndromes $[13,14]$. A summary of mostly common used Chinese herbal medicines in SLE [15] and the characteristics was listed in Table 1 . The former mainly is called heat toxin syndrome and is characterized by the fresh red skin rash, severe joint pain, multiple oral ulcers, high fever, dark colored urine, crimson tongue with yellow coating and rapid 
TABLE 1: Comparison of different Chinese herbal medicines in SLE.

\begin{tabular}{|c|c|c|c|}
\hline Chinese name & Full scientific name & Action in TCM & Immunomodulatory effects \\
\hline Ren Shen & Radix Ginseng & $\begin{array}{l}\text { Replenishes } q i \text { and strengthen yang } \\
\text { Tonify the spleen and lungs }\end{array}$ & $\begin{array}{l}\text { Stimulates the bone marrow and improves } \\
\text { the phagocytic function of monocytes }\end{array}$ \\
\hline Huang Qi & Radix Astragali & Tonify spleen and lung $q i$, and wei $q i$ & $\begin{array}{l}\text { Enhancement of T-cell proliferation, } \\
\text { promoting NK cell killing effects }\end{array}$ \\
\hline Gou Qi & Wolfberry Fruit & $\begin{array}{l}\text { Nourishes liver and kidney and tonifies } \\
\text { yin }\end{array}$ & $\begin{array}{l}\text { Activates the thymus cell proliferation and } \\
\text { improves IL- } 2 \text { and white blood cells }\end{array}$ \\
\hline He Shou Wu & Radix Polygoni Multiflori & Nourishes kidney and blood & $\begin{array}{l}\text { Enhancement of C3b receptor, promoting } \\
\text { interferons (IFNs) production }\end{array}$ \\
\hline Ling Zhi & Ganoderma Lucidum & Boosts heart qi and calms shen & $\begin{array}{l}\text { Inhibits platelet aggregation and improves } \\
\mathrm{CD}^{4+} \text { level and } \mathrm{CD}^{4+} / \mathrm{CD}^{8+} \text { level }\end{array}$ \\
\hline Gan Cao & Glycyrrhiza Uralensis & Tonifies $q i$ and clears heat and toxic fire & $\begin{array}{l}\text { Modulates serum level of IgE, IgA, and IgM } \\
\text { antibodies, decreases serum level TNF- } \alpha \text {, } \\
\text { and induces B cell maturation }\end{array}$ \\
\hline
\end{tabular}

sunken pulse [16]. The latter has manifestations as deficiency of Qi and Yin with benign skin rash, minor joint pain, lack of strength, shortness of breath, dry throat and mouth, vexing thirst, small amount of urine, red tongue with dry and/or few coating, and rapid string-like pulse or rapid fine pulse [17]. Therapeutic principles and practices vary accordingly. The formula Zi Shen Qing (ZSQ) was originated from classic CM formulae Yu Ping Feng San and comprised of six herbs (Table 2). Through the combination of these herbs, ZSQ can tonify Qi, enrich Yin, remove internal heat, and clear the toxin [18].

Our research team has studied ZSQ for SLE from 1990s. We found that ZSQ could normalize the low C3 and C4 as well as reverse anti-dsDNA positivity [19]. It also provided reduction of corticosteroid dosage and prevention of severe disease flare [20, 21]. Previous studies had many limitations, for example, proper randomization procedures were absent, blinding was inadequate, strict eligible criteria (including Western Medicine and Chinese Medicine Syndrome) were not clearly defined, quality control was not conducted, and so forth [17-21]. To further assess the effectiveness and safety of ZSQ in the mild-to-moderate SLE with "deficiency of Qi and Yin" pattern, well-controlled granules and standard clinical parameters were performed in this pilot clinical trial.

\section{Methods}

2.1. Participants and Setting. All the participants were recruited via advertisements in local newspapers and outpatient specialist clinics of rheumatology in LongHua Hospital, Shanghai, China, from 1st of March 2006 to 31st of January 2009 for preliminary screening. Preliminary telephone interviews were conducted to determine whether potential participants met the broad inclusion criteria. Participants were then screened by a rheumatologist to ensure their medical suitability before entering into the trial. Diagnosis of SLE was based on 1997 American College of Rheumatology (ACR) classification criteria for SLE [22]. Diagnosis of "deficiency of Qi and Yin" SLE was based on the criteria described in Guidance for Clinical Research of Chinese Herbal Medicine 2002 and Therapeutic Effect of Disease and Syndromes in Traditional
Chinese Medicine [23, 24]. All participants presented with any three of the chief symptoms and any two of the simultaneous symptoms had diagnosis of “deficiency of Qi and Yin”. Chief symptoms included (i) red skin rashes; (ii) dry mouth and oral ulcers; (iii) muscle or joint pain; (iv) vexing heat in the body; (v) red tongue with dry and/or few coating; (vi) rapid string-like pulse or rapid fine pulse. Simultaneous symptoms included (i) low back pain and lack of strength; (ii) dry mouth and eyes; (iii) tinnitus; (vi) hair loss.

Participants were recruited only from those who (i) age 12-65 years old; (ii) having diagnosis of SLE; (iii) have total scores of SLEDAI-2000 from 5 to 14 indicating mild-tomoderate disease activity; (vi) have diagnosis of "Deficiency of Qi and Yin" CM syndrome; (v) have stable treatment regimen for at least 30 days with prednisone alone (5-30 mg/day) or combined with nonsteroidal anti-inflammatory drugs; (vi) have seropositivity as defined by 2 positive ANA or antidsDNA test results (ANA titer $\geqq 1: 80$ and/or anti-dsDNA antibody level $\geqq 30 \mathrm{IU} / \mathrm{mL}$ ), of which $\geqq 1$ test result had to be obtained during screening. Exclusion criteria included serious intercurrent illness, severe active lupus nephritis, severe central nervous system manifestations, CHM allergies, severe psychiatric disease, and pregnancy. The study was conducted in accordance with the Declaration of Helsinki and was approved by the Institutional Review Board of Shanghai University of Traditional Chinese Medicine/LongHua Hospital. All participants gave their written, informed consent and were free to withdraw at any time from the trial.

2.2. Study Design. The study consisted of a 2 -week baseline period and 12-week treatment period. Eligibility screening was performed, including questionnaires of SLE CM syndrome scores; SLEDAI-2000 and information on demographic characteristics, medications, smoking, and alcohol consumption were also collected. After the eligible screening visit, participants entered a 2 -week run-in phase to obtain baseline data. These consisted of SLEDAI scores, whole blood count, liver and renal function tests, immunologic index as C3, C4, IgG, ANA, anti-dsDNA, NK, and sIL-2R. Two blood samples were obtained at baseline and endpoint by our research nurse and checked by the Medical Laboratory, 
TABLE 2: Composition and function of ZSQ granules.

\begin{tabular}{|c|c|c|c|c|c|c|c|}
\hline Chinese name & Full scientific name & Parts of plant use & $\%$ & $\begin{array}{l}\text { Dose of } \\
\text { dry plant }\end{array}$ & Constituent & $\begin{array}{l}\text { Dose after } \\
\text { extraction }\end{array}$ & Function in Chinese medicine \\
\hline Huang Qi & Radix Astragali & Dry root & & 15 & Astragaloside & 3.75 & Tonifies Qi \\
\hline Sheng $D i$ & $\begin{array}{l}\text { Rehmannia } \\
\text { glutinosa Libosch }\end{array}$ & Dry root & & 15 & & 3.75 & Nourishes Yin \\
\hline Shan Zhu Yu & Fructus Cornus & Dry fruit & & 5 & Loganin & 1.25 & $\begin{array}{l}\text { Tonifies the liver and kidney and } \\
\text { retains the essence }\end{array}$ \\
\hline Bai Shao & Paeonia lactiflora & Dry root & & 15 & Paeoniflorin & 3.75 & $\begin{array}{l}\text { Nourishes blood and preserves } \\
\text { Yin }\end{array}$ \\
\hline Dan Pi & $\begin{array}{l}\text { Cortex Moutan } \\
\text { Radicis }\end{array}$ & Dry root and stem & & 15 & Paeonol & 3.75 & Clears deficient fire and heat \\
\hline She She Cao & $\begin{array}{l}\text { Herba Hedyotidis } \\
\text { Diffusae }\end{array}$ & Dry leaf & & 15 & Oleanic acid & 3.75 & Clears heat and resolves toxins \\
\hline Total & & & 100 & 80 & & 20 & \\
\hline
\end{tabular}

LongHua Hospital, Shanghai University of TCM. These data were used to ensure the minimum eligibility criteria for each participant and to screen out respondents with potential poor compliance. After the run-in phase, patients who met entry criteria were randomly assigned to either the ZSQ group or the controlled group. Hydroxychloroquine (HCQ) sulfate tablet (Shanghai Zhongxi Pharmaceutical Co., Ltd.) was used as the controlled medication, which was prescribed as $100 \mathrm{mg}$ twice a day. We have a standard plan for glucocorticoids (GC) according to Kelley's Textbook of rheumatology, 8th edition [6]. Low-dose GC therapy ( $\leq 7.5 \mathrm{mg}$ prednisone equivalent per day) was generally used to control mild SLE activity. In moderate SLE, GCs were used as either single or background therapy in combination with immunosuppressive agents, at doses of 0.5 to $1 \mathrm{mg} / \mathrm{kg}$ prednisone equivalent in a single dose usually in the morning. When combined with immunosuppressive agents, the GC dose should rarely exceed 0.5 to $0.6 \mathrm{mg} / \mathrm{kg}$ prednisone due to concerns for infections and other toxicities. Tapering of GC dose started after the first 4 to 6 weeks of therapy, targeting a dose of $0.25 \mathrm{mg} / \mathrm{kg}$ every other day at 2 to 3 months, which was acceptable for long-term use.

Consultations were conducted every 4 weeks over the 12 week treatment period. To maintain blinding, the investigators were not allowed to ask the participants about the types of the interventions, and the participants were instructed not to reveal their interventions to the investigators and discuss, related issues with them. Every participant was given a 4-week supply of medication at each treatment visit by the pharmacists who had no connection to the research. In addition, responses to the SLEDAI and CM syndrome scores were assessed by independent assessor who was also blinded to patients' interventions. Compliance was controlled by counting the remaining sachets. A participant was considered compliant if she/he consumed not less than $80 \%$ of the daily dose of the study medication.

\subsection{Interventions}

2.3.1. CHM Intervention. ZSQ is composed of Radix astragali, Rehmannia glutinosa libosch, Fructus Corni, Paeonia lactiflora, Herba Hedyotis Diffusae, and Cortex Moutan Radicis.
The composition and action of each herb is summarized in Table 2. Patients are instructed to dissolve a sachet of granules $(10 \mathrm{~g})$ in $200 \mathrm{~mL}$ of hot water; they take this solution orally twice daily for 12 weeks. The preparation of herbs and methods of quality control had been published in our previous paper $[25,26]$ and listed in Supplement 1 (see supplementary material available at http://dx.doi.org/10.1155/2013/327245) About one gram of three batches granula ZSQ (Lot. 060611, 060612, 060613) was, respectively, extracted by about $9 \mathrm{~mL}$ methanol for $20 \mathrm{~min}$ under ultrasound, and then methanol was added till the total volume was $10 \mathrm{~mL}$. After filtration, supernatant was centrifuged at $10,000 \mathrm{r} / \mathrm{min}$ for $10 \mathrm{~min}$ and filtered through $0.22 \mu \mathrm{m}$ PTFE syringe filter. Filtrate was diluted 10 times with methanol, and an aliquot of dilution $(20 \mu \mathrm{L})$ was injected into HPLC-DAD analysis.

2.3.2. WM Intervention. Fen Le tablet is selected as the active control, for which each tablet consists of $100 \mathrm{mg}$ hydroxychloroquine sulfate. It was manufactured by Shanghai Zhongxi Pharmaceutical Co., Ltd (http://www.shzxyy.com/ English/index.asp). Patients were instructed to take 1 tablet twice daily for 12 weeks.

All ZSQ granules were prepared by Xiu Long Pharmaceutical (Shanghai.) Co. Limited. The entire manufacturing process, from authenticating the raw materials to the final products, is in strict compliance with the standards of Good Manufactory Practice (GMP) and Chinese Pharmacopoeia [15]. Acute toxicity test of ZSQ granules on mice had been done for monitoring the toxicity of this formula. The results showed that maximum tolerance dosage was $45 \mathrm{~g} / \mathrm{kg}$, equal to 150 times of the dose tested. The chemical compositions of the final products are analyzed, while contamination with heavy metals, toxic elements, microbes, and pesticide residue was tested by an SGS laboratory. The ZSQ granules were packed in sealed opaque aluminium sachets and put in zip lock bag (28 sachets each), while WM intervention was put in a plastic bottle (56 tablets each).

2.4. Outcome Measures. The primary outcome was the changes of the SLEDAI-2000 [27]. SLEDAI was originally 
developed and validated as a clinical index for the measurement of disease activity in SLE and has been used as a global measurement of disease activity in SLE since 1985 and modified by a panel of experienced rheumatologists with expertise in SLE, using well-established group techniques and index development methodology in 2000. The index has been used by both researchers and clinicians and also is shown to be valuable as a predictor for mortality [28-30].

The secondary outcomes included score changes of Chinese medicine syndromes (CMSs), the changes of steroid dosage, and the incidence of lupus flares. The items of CMS and description of rating were listed in Table 3. Mildto-moderate flares were defined as one or more of the following features: (1) a $>3$-point change in the SLEDAI score, with a total score of $\leq 12$; (2) new or worsening discoid, photosensitivity, or other rash attributable to lupus, nasopharyngeal ulcers, pleuritis, pericarditis, arthritis, or fever not attributable to infection; (3) an increase in the prednisone dosage, but not to $>0.5 \mathrm{mg} / \mathrm{kg}$ of body weight per day. Severe flares were defined as (1) an SLEDAI score of $>12$; (2) new or worsening central nervous system (CNS) involvement, vasculitis, glomerulonephritis, myositis, thrombocytopenia (platelet count $<60 \times 10^{9}$ cells/liter), or hemolytic anemia (hemoglobin level $<70 \mathrm{gm} /$ liter or a decrease in the hemoglobin level of $>30 \mathrm{gm} /$ liter over a 2 -week period), each of which required doubling of the corticosteroid dosage to a final dosage of $>0.5 \mathrm{mg} / \mathrm{kg} /$ day or hospitalization; (3) any manifestation requiring an increase in the dosage of prednisone or to $>0.5 \mathrm{mg} / \mathrm{kg} /$ day [31]. Assessed biologic markers included serums IgG, C3, C4, anti-dsDNA, ANA, $\mathrm{NK}$, and sIL-2R. Serologic assessments were conducted using enzyme-linked immunosorbent assay, except for ANA.

2.5. Randomization and Blinding. A random sequence was prepared by staff at the GCP center of Longhua Hospital, with no connection to the study. Block randomization was carried out in a 1:1 ratio according to the sequence generated with Random Allocation Software (version 1.0.0), Isfahan, Iran. Randomization codes were kept in sealed, opaque envelopes labeled with consecutive random numbers and kept in a location far away from the clinical setting where participants were assessed. Emergency envelopes with the randomization codes were kept by principle investigator. Treatment assignments were not revealed to all the investigators (including outcome assessor), until the study was completed. The investigators, nurses, all other participating medical staff, and the assessors remained unaware of intervention assignments throughout the trial. Only the participants and pharmacists knew the intervention and were instructed not to tell the investigators/assessors about the intervention. Assessment of the success of blinding to the investigators was conducted at the end of the trial.

2.6. Sample Size Calculation. The sample size calculation was based on the primary outcome measure. Previous studies of hydroxychloroquine (HCQ) sulfate tablet in the reduction of SLEDAI were about 30\% [32]. Our pilot observational study findings were consistent with a reported double-blinded clinical study of CHM on SLE, in that at least $15 \%$ reduction in SLEDAI was reported in the CHM group compared to the control [33]. We assumed a difference of at least 15\% between ZSQ and HCQ. The sample size in each group was determined by using the formula derived by Whitley and Ball [34]:

$$
n=\frac{\left[p_{1}\left(1-p_{1}\right)+p_{2}\left(1-p_{2}\right)\right]}{\left(p_{1}-p_{2}\right)^{2}} \times C_{p, \text { power }} .
$$

When type I: $\alpha=0.05, \beta=0.20$; power $(1-\beta)=80 \%$; $p_{1}=30 \%, p_{2}=45 \%$ and when two-tailed level $\alpha$ and power were set at 0.05 and $80 \%$, respectively. 35 participants were needed in each group. Estimating about 20\% dropouts over 8 weeks of treatment, the number of each group was 42 , and a total of 84 participants were needed for this study.

2.7. Statistical Analysis. All efficacy and safety analyses were conducted according to the intention-to-treat (ITT) principle. Missing values were imputed by the last-observationcarried-forward method. The statistical analysis was performed using the Statistical Packages of Social Sciences (SPSS) for Windows version 16.0. The statistical significance was defined as two-sided $P$ value of $<0.05$. Baseline characteristics were reported as mean (SD), frequency, and percentages. Baseline differences between the groups were assessed with the use of Student's $t$-test for normally distributed continuous variables and nonparametric Mann-Whitney $U$ test for nonnormally distributed. For categorical variables, chi-squared test or Fisher's exact test were used. Comparisons between groups were conducted by using an analysis of covariance (ANCOVA) with baseline as covariate. All items of CMS were compared between treatment groups for each 4 week using ANCOVA, with treatment group as a factor in the model and baseline as the covariate. The change from baseline to 12 weeks in score of SLEDAI and CMS was tested with repeated measure analysis of variance (ANOVA). Within-group differences were assessed with paired $t$-test for normally distributed data and Wilcoxon signed-rank test for nonnormally distributed data. For biological markers and safety indices, ANCOVA was used to compare difference between two groups, adjusting for baseline covariates.

\section{Results}

3.1. Participant Flow. The participant flow was listed in Figure 1. In total, 203 Chinese SLE patients were recruited from 1st of March 2006 to 31st of January 2009 for preliminary screening. 106 participated in baseline eligibility screening for randomization. 12 participants were screened out due to abnormal liver function, 7 participants were screened out due to abnormal white blood cell numbers, and 3 were screened out due to intercurrent illness. A total of 7 participants (4 from the ZSQ group and 3 from the control group) withdrew during the study period; reasons were listed in the flow diagram.

3.2. Baseline Data. The baseline characteristics of the participants were summarized in Table 4 . The mean age of ZSQ 
TABLE 3: Chinese medicine syndromes rating scales.

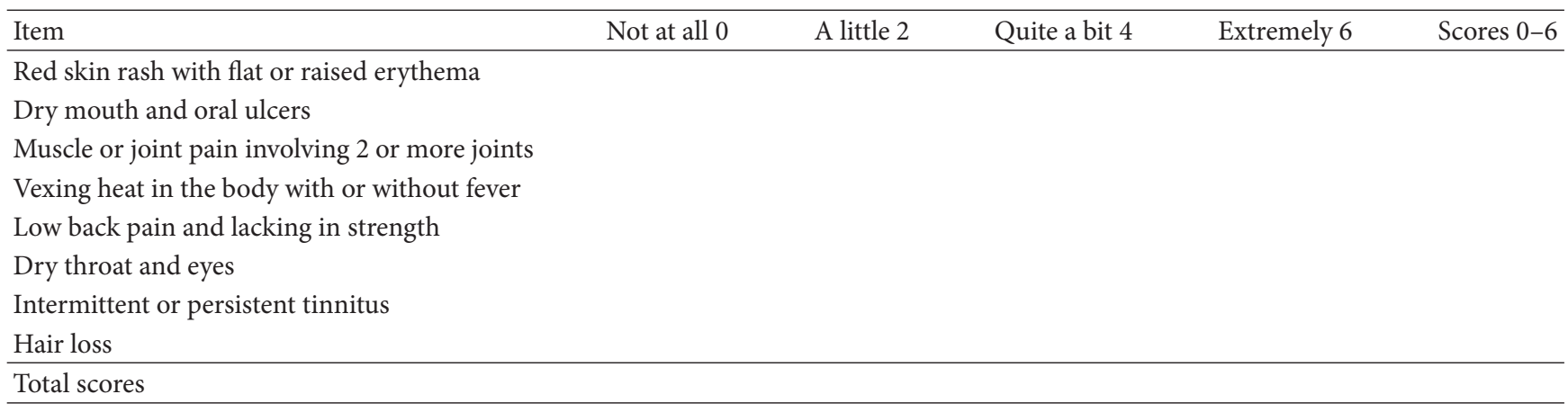

TABLE 4: Baseline demographic and clinical characteristics of the patients.

\begin{tabular}{|c|c|c|c|}
\hline Characteristic & ZSQ group $(n=42)$ & Control group $(n=42)$ & $P$ value \\
\hline Age, yr & $32.7 \pm 13.6$ & $34.8 \pm 14.3$ & 0.46 \\
\hline Education, yr & $10.5 \pm 3.7$ & $10.6 \pm 3.9$ & 0.35 \\
\hline BMI, $\mathrm{kg} / \mathrm{m}^{2}$ & $22.1 \pm 2.6$ & $22.7 \pm 3.1$ & 0.44 \\
\hline Female $(n, \%)$ & $39(92.8 \%)$ & $38(90.48 \%)$ & 0.67 \\
\hline Disease duration, yr & $4.7 \pm 4.4$ & $5.0 \pm 4.6$ & 0.79 \\
\hline Dosage of prednisone, $\mathrm{mg} / \mathrm{d}$ & $10.2 \pm 6.4$ & $10.6 \pm 6.6$ & 0.62 \\
\hline SLEDAI scores & $10.5 \pm 2.2$ & $10.7 \pm 2.1$ & 0.51 \\
\hline \multicolumn{4}{|l|}{ SLE manifestations $(n, \%)$} \\
\hline Nephritis & $32(76.2 \%)$ & $34(81.0 \%)$ & \multirow{7}{*}{$>0.05$} \\
\hline Arthritis & $20(47.6 \%)$ & $18(42.9 \%)$ & \\
\hline Dermatitis & $24(57.1 \%)$ & $21(50.0 \%)$ & \\
\hline Central nervous system & $2(4.8 \%)$ & $1(2.4 \%)$ & \\
\hline Lymphopenia & $9(21.4 \%)$ & $8(19.0 \%)$ & \\
\hline Thrombocytopenia & $6(14.3 \%)$ & $7(16.7 \%)$ & \\
\hline Pericarditis & $1(2.3 \%)$ & $0(0.0 \%)$ & \\
\hline Total scores of CMS & $19.5 \pm 3.9$ & $19.7 \pm 3.9$ & 0.80 \\
\hline Anti-dsDNA (IU/mL) & $89.8 \pm 72.9$ & $89.2 \pm 80.5$ & 0.63 \\
\hline $\mathrm{C}_{3}(\mathrm{~g} / \mathrm{L})$ & $0.8 \pm 0.3$ & $0.8 \pm 0.2$ & 0.47 \\
\hline $\mathrm{C}_{4}(\mathrm{~g} / \mathrm{L})$ & $0.2 \pm 0.1$ & $0.3 \pm 0.1$ & 0.61 \\
\hline $\operatorname{IgG}(\mathrm{g} / \mathrm{L})$ & $18.7 \pm 5.4$ & $17.9 \pm 2.8$ & 0.38 \\
\hline NK cell activity (\%) & $14.5 \pm 8.8$ & $14.0 \pm 6.2$ & 0.78 \\
\hline sIL-2R (pg/mL, PM) & $40.8 \pm 8.6$ & $40.1 \pm 14.8$ & 0.80 \\
\hline
\end{tabular}

group was $32.7 \pm 13.6$ years, and the mean age of control group was $34.8 \pm 14.3$ years $(P=0.46)$. The mean prednisone dosage of ZSQ group was $10.2 \pm 6.4 \mathrm{mg} / \mathrm{d}$, and control group was $10.6 \pm 6.6$, respectively $(P=0.62)$. The disease duration was $4.7 \pm 4.4$ months in ZSQ group and $5.0 \pm 4.6$ months in control group $(P=0.79)$. The baseline characteristics of the two groups were balanced $(P>0.05)$.

3.3. Primary Outcome Assessment. The total scores of SLEDAI decreased from $10.5 \pm 2.2$ to $5.1 \pm 1.5$ in the ZSQ group and from $10.6 \pm 2.1$ to $7.0 \pm 1.9$ in the control group, during the 12 -week treatment period ( $P=0.03$; Table 5$)$.

3.4. Secondary Outcome Assessment. The total scores of CMS decreased from $19.5 \pm 3.9$ to $12.3 \pm 4.3$ in the ZSQ group and from $19.7 \pm 3.9$ to $14.6 \pm 5.2$ in the control group, during the 12 -week treatment period ( $P=0.04$; Table 5$)$. The mean prednisone dosage decreased from $10.2 \pm 6.4$ to $3.6 \pm 3.1$ in the ZSQ group and from $10.6 \pm 6.6$ to $6.6 \pm 5.6$ in the control group during the 12 -week treatment period $(P=$ 0.01; Table 5). The incidence of mild-to-moderate flare-up was $11.9 \%$ in the ZSQ group and $19.0 \%$ in the control group after the 12-week treatment period based on SLE Flare Index $(P=0.02$; Table 5$)$, and there was no incidence of severe flareup in both of the groups.

3.5. Biologic Markers. ZSQ treatment produced sustained reductions in anti-dsDNA and IgG level as well as increased $\mathrm{C} 3$ and $\mathrm{C} 4$ concentrations, compared with control group $(P<$ 0.05 , Table 5$)$. The NK cell activity was increased from (14.5 \pm $8.8) \%$ to $(16.7 \pm 9.1) \%$ in ZSQ group and from $(14.0 \pm 6.2) \%$ to $(16.3 \pm 4.2) \%$ as well as increased the level of sIL-2R from $(40.8 \pm 8.6) \mathrm{pM}$ to $(37.2 \pm 12.5) \mathrm{pM}$ in ZSQ group and from $(40.1 \pm 14.8) \mathrm{pM}$ to $(38.6 \pm 13.8) \mathrm{pM}$ in control group, but there is no significance between the two groups $(P>0.05)$. 
TABLE 5: Comparison of the main outcome measures within and between ZSQ and control groups.

\begin{tabular}{|c|c|c|c|c|c|c|c|}
\hline \multirow[b]{2}{*}{ Outcome measures } & \multicolumn{3}{|c|}{ ZSQ $(n=42)$} & \multicolumn{4}{|c|}{ Control $(n=42)$} \\
\hline & Mean & $\mathrm{SD}$ & $\begin{array}{l}\text { Within-group } \\
P \text { value }^{\text {a }}\end{array}$ & Mean & SD & $\begin{array}{l}\text { Within-group } \\
P \text { value }^{\text {a }}\end{array}$ & $\begin{array}{c}\text { Between-group } \\
\quad P \text { value } \\
\end{array}$ \\
\hline \multicolumn{8}{|l|}{ SLEDAI scores } \\
\hline Baseline & 10.5 & 2.2 & & 10.6 & 2.1 & & 0.35 \\
\hline Endpoint (12-week) & 5.1 & 1.5 & $<0.01$ & 7.0 & 1.9 & $<0.01$ & 0.03 \\
\hline \multicolumn{8}{|l|}{ CMS scores } \\
\hline Baseline & 19.5 & 3.9 & & 19.7 & 3.9 & & 0.80 \\
\hline Endpoint (12-week) & 12.3 & 4.3 & $<0.01$ & 14.6 & 5.2 & $<0.01$ & 0.04 \\
\hline \multicolumn{8}{|l|}{ Mean prednisone dosage } \\
\hline Baseline & 10.2 & 6.4 & & 11.0 & 6.6 & & 0.39 \\
\hline Endpoint (12-week) & 3.6 & 3.1 & $<0.01$ & 6.6 & 5.6 & $<0.01$ & 0.01 \\
\hline $\begin{array}{l}\text { Mild-to-moderate flare-up at } \\
\text { endpoint }(n, \%)\end{array}$ & $5(11.9)$ & & & $8(19.0)$ & & & 0.02 \\
\hline Severe flare-up & $0(0.0)$ & & & $0(0.0)$ & & & \\
\hline \multicolumn{8}{|l|}{ Anti-dsDNA (IU/mL) } \\
\hline Baseline & 89.8 & 72.9 & & 89.2 & 80.5 & & 0.63 \\
\hline Endpoint (12-week) & 76.3 & 61.6 & $<0.01$ & 83.4 & 72.6 & 0.08 & $<0.01$ \\
\hline \multicolumn{8}{|l|}{$\mathrm{C}_{3}(\mathrm{~g} / \mathrm{L})$} \\
\hline Baseline & 0.8 & 0.3 & & 0.8 & 0.2 & & 0.47 \\
\hline Endpoint (12-week) & 1.2 & 0.3 & 0.02 & 0.8 & 0.3 & 0.27 & 0.02 \\
\hline \multicolumn{8}{|l|}{$\mathrm{C}_{4}(\mathrm{~g} / \mathrm{L})$} \\
\hline Baseline & 0.2 & 0.1 & & 0.3 & 0.1 & & 0.61 \\
\hline Endpoint (12-week) & 0.3 & 0.2 & 0.04 & 0.2 & 0.1 & 0.27 & 0.04 \\
\hline \multicolumn{8}{|l|}{$\operatorname{IgG}(\mathrm{g} / \mathrm{L})$} \\
\hline Baseline & 18.7 & 5.4 & & 17.9 & 2.8 & & 0.38 \\
\hline Endpoint (12-week) & 17.0 & 5.0 & $<0.01$ & 17.8 & 3.2 & 0.66 & 0.03 \\
\hline \multicolumn{8}{|l|}{ NK cell activity (\%) } \\
\hline Baseline & 14.5 & 8.8 & & 14.0 & 6.2 & & 0.78 \\
\hline Endpoint (12-week) & 16.7 & 9.1 & $<0.01$ & 16.3 & 4.2 & $<0.01$ & 0.29 \\
\hline \multicolumn{8}{|l|}{ sIL-2R (pM) } \\
\hline Baseline & 40.8 & 8.6 & & 40.1 & 14.8 & & 0.80 \\
\hline Endpoint (12-week) & 37.2 & 12.5 & $<0.01$ & 38.6 & 13.8 & $<0.01$ & 0.11 \\
\hline
\end{tabular}

${ }^{a}$ Results from paired $t$-test to examine the effects of within-group, baseline versus 12-week, and 12-week versus 3-month followup.

${ }^{b}$ Results from an analysis of covariance (ANCOVA) with baseline as covariate, ZSQ group versus control group.

TABLE 6: Summary of adverse events.

\begin{tabular}{lcc}
\hline Items & $\begin{array}{c}\text { ZSQ group }(n=42), \\
\text { no. }(\%)\end{array}$ & $\begin{array}{c}\text { Control group }(n=42) \\
\text { no. }(\%)^{\mathrm{a}}\end{array}$ \\
\hline Headache & $6(14.3)$ & $4(9.5)$ \\
Nausea & $2(4.8)$ & $1(2.4)$ \\
Diarrhea & $6(14.3)$ & $4(9.5)$ \\
Insomnia & $5(11.9)$ & $2(4.8)$ \\
Dry skin & $5(11.9)$ & $8(19.0)$ \\
Epigastric pain & $3(7.1)$ & $3(7.1)$ \\
\hline
\end{tabular}

${ }^{a} P$ value was calculated from Fisher's exact test and $P>0.05$.

3.6. Safety and Adverse Events. Whole blood count, renal and liver functions, urine test, and EKG were checked at baseline and after treatments. There were no differences between the two groups in these safety indices. During the study, very few patients experienced slightly adverse events including headache, dry skin, diarrhea, insomnia, and irregular menstrual period and so forth (Table 6).

3.7. Assessment of Blinding and Compliance. Success of blinding was evaluated by asking the investigators which treatment they thought patients had received at each consultation. Overall the investigators identified $45.2 \%$ correctly of their treatment (38 among 84 patients), whether ZSQ or control medications.

Compliance at 12-week treatment was fair with three withdrawn in control group and four withdrawn in ZSQ group (Figure 1). The overall compliance with medication 


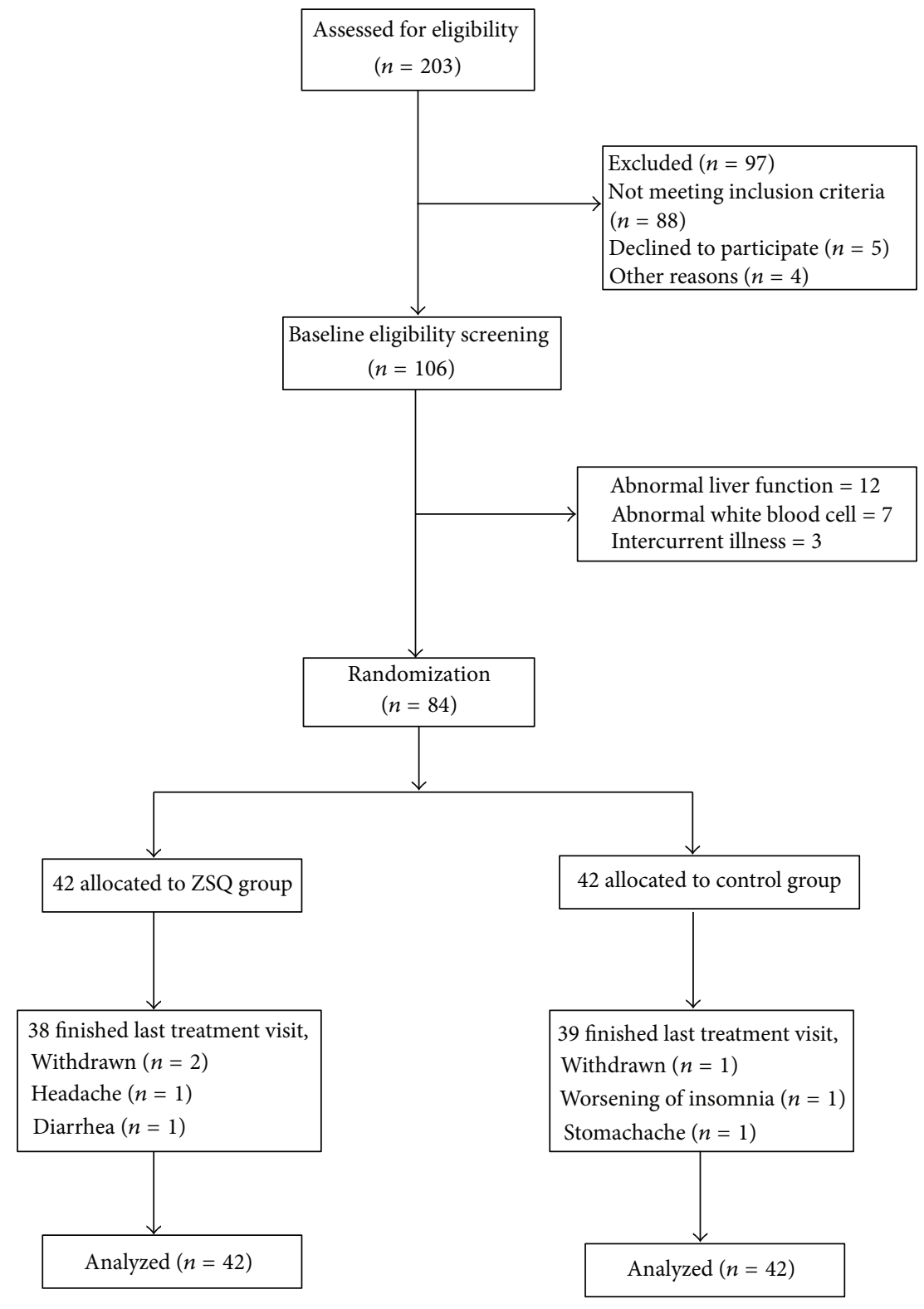

FIgURe 1: Participant flow diagram.

in the remaining participants was good. $92 \%$ of participants were found to have taken at least $80 \%$ of scheduled doses, as determined by counting remaining medication sachets.

\section{Discussion}

In this pilot prospective, single-blinded, and randomized controlled study, we found that ZSQ was superior over controlled group in decreasing the total scores of SLEDAI2000 and CMS, and effectively reducing steroid dosage as well as the frequency of disease flare-up. In the specific domains of "deficiency of Qi and Yin," ZSQ can also relieve the symptoms of dry mouth, hair loss, and muscle or joint pain. Moreover, the sustained benefits of ZSQ were observed in the follow-up period.
The choice of the positive control not the placebo control is mainly based on the ethical consideration. Large scales of clinical studies had shown that SLE was mostly like flare when using low dose of corticosteroids alone [35]. Antimalarial drugs, mainly chloroquine (CQ) and HCQ, are commonly prescribed to SLE patients with skin and joint manifestations and are increasingly identified as an adjuvant treatment for achieving remission in severe lupus [36, 37]. Recent studies also indicated that it could prevent irreversible organ damage [38]. The adverse effects of antimalarial drugs included retinopathy, myopathy, and minor myocardiopathy and so forth. In addition, these drugs had antiplatelet and hypolipidemic effects. In the setting of systemic autoimmune diseases, like SLE, antimalarial drugs act upon a panoply of targets without increasing risk of opportunistic infections. 
ZSQ comprised of Radix Astragali, Rehmannia glutinosa Libosch, Fructus Corni, Rhizoma Atractylodis Macrocephalae, Herba Hedyotis Diffusae, and Cortex Moutan Radicis. It originates from the classic formula "Yu Ping Feng San (YPFS)," and it has been commonly used to regulate the immune systems [39]. In the study of healthy volunteers, CD4/CD8 ratio of T-lymphocytes was significantly increased after taking the YPFS regimen for 14 days, and no such increase was observed after the consumption was stopped (day 29) as well as during the entire control study period [40]. Our animal study also indicated that three different dosages of ZSQ granules (low dosage of $1.6 \mathrm{~g} / \mathrm{kg} /$ day, moderate dosage of $3.2 \mathrm{~g} / \mathrm{kg} / \mathrm{day}$, high dosage of $6.4 \mathrm{~g} / \mathrm{kg} /$ day) would increase the serum level of total white blood cells, and only moderate and high dosage groups would increase the levels of CD3, CD8, and CD4 [41]. In our study, we also found that the concentration level of $\mathrm{C} 3$ and $\mathrm{C} 4$ was increased, which plays a central role in the activation of complement system. Complement activation is an efficient means to coat antigens so that they adhere to peripheral blood cells and are then phagocytosed or carried to the spleen and liver for disposal $[41,42]$. In these organs, the immune complexes are transferred to tissue macrophages for destruction or an immune response. Complement measurements are helpful in diagnosing and following patients with systemic lupus erythematosus. Low C4 and C3 in the presence of antidouble-stranded antibodies have nearly a $100 \%$ specificity for lupus [43].

The beneficial roles of NK cells are involved in immune tolerance and can ameliorate or prevent tissue inflammation. In SLE patients, the reduced proportion and function of NK cells correlate with disease activity, and NK cells correlate inversely with IgG levels [44]. In current study, we found that the results were consistent with previous study, and ZSQ increased the NK cell activity and decreased the IgG level compared with control group [45]. In SLE patients, it has been found that activated $\mathrm{T}$ cells and $\mathrm{B}$ cells release both interleukin-2 and a soluble form of interleukin-2 receptor (sIL-2R) [46]. The serum IL-2R level thus has been used as a marker for disease activity [47]. In this study, we found that the level of sIL-2R in ZSQ group and in control group increased after treatment, but there is no significance between the two groups.

The corticosteroids exert broad inhibitory effects on immune responses mediated by $\mathrm{T}$ and $\mathrm{B}$ cells, and their rapid onset of actions made them widely used in managing acute SLE manifestations [48-50]. However, the adverse effects involve early (mood effects, acne, myalgias, and infections); later (metabolic); late-onset (osteoporosis, avascular bone necrosis, cataract, and cardiovascular disease) events [51]. Furthermore, about $30 \%$ patients responded relapse when steroids are withdrawn and become steroid dependent [52]. So reduction to the dose of corticosteroid and induction of the remission of SLE are of paramount importance. ZSQ was investigated in combination with corticosteroids in this pilot study, and it was superior over controlled group in reducing the dosage of corticosteroids. The incidence of lupus flares in ZSQ group was $11.9 \%$ and in control group was $19.0 \%$, respectively, which may indicate the preventive effects of SLE flare-ups in ZSQ group.

Our study has several limitations. First, it was a singleblinded clinical trial without a placebo group, and it may affect the patients' response to the assessments. Although a double-blinded, placebo-controlled trial would have been optimal, we believe that the investigators were effectively blinded because the patients were instructed not to reveal the interventions and the assessment of blinding was successful. Second, self-selection bias is likely present, as most of the study subjects were recruited through newspaper advertisements and agreed to participate in this clinical trial. Most of them expressed negative views of immunosuppressive agents and corticosteroids, during intake interviews, and many may even have had high prior expectations regarding the effectiveness of Chinese medicine. Such expectations may have led to inaccuracies in reporting symptoms. Third, our study was a small, single center trial in a single ethnic Chinese population, and thus, findings may not be broadly generalizable. Although our findings indicated that ZSQ was superior to HCQ in some outcome measurements, the tentatively positive effects shown in the present study should be interpreted with caution.

In conclusion, our study shows that the Chinese herbal medicine ZSQ is effective and in the mild-to-moderate Chinese SLE patients with "deficiency of Qi and Yin" pattern in reducing SLE disease activity and withdrawal dosage of corticosteroids. It is safe and well tolerated, with no serious adverse events noted during the study period. Larger-scales, multicenter, active controlled and/or placebo-controlled, clinical studies of ZSQ on more diverse populations are needed to further confirm these findings.

\section{Conflict of Interests}

The authors declare that they have no conflict of interests.

\section{Acknowledgment}

This work was supported by the Bureau of Health, Shanghai Government: A pilot study on the effects and mechanism of a Chinese herbal formula on Systematic Lupus Erythematosus (Project no.: 2004X001A) and Longhua Medical Project (LYTD-14).

\section{References}

[1] T. Stoll, N. Sutcliffe, J. Mach, R. Klaghofer, and D. A. Isenberg, "Analysis of the relationship between disease activity and damage in patients with systemic lupus erythematosus: a 5-year prospective study," Rheumatology, vol. 43, no. 8, pp. 1039-1044, 2004.

[2] American College of Rheumatology Ad Hoc Committee on Systemic Lupus Erythematosus Guidelines, "Guidelines for referral and management of systemiclupus erythematosus in adults," Arthritis \& Rheumatism, vol. 42, no. 9, pp. 1785-1796, 1999.

[3] J. Nossent, E. Kiss, B. Rozman et al., "Disease activity and damage accrual during the early disease course in a multinational 
inception cohort of patients with systemic lupus erythematosus," Lupus, vol. 19, no. 8, pp. 949-956, 2010.

[4] J. Klippel, Primer on the Rheumatic Diseases, Arthritis Foundation, Atlanta, Ga, USA, 11th edition, 1997.

[5] J. Font, R. Cervera, M. Ramos-Casals et al., "Clusters of clinical and immunologic features in systemic lupus erythematosus: analysis of 600 patients from a single center," Seminars in Arthritis and Rheumatism, vol. 33, no. 4, pp. 217-230, 2004.

[6] G. S. Firestein, Kelley's Textbook of Rheumatologyed, Saunders, Philadelphia, Pa, USA, 8th edition, 2008.

[7] A. Mak, D. A. Isenberg, and C. S. Lau, "Global trends, potential mechanisms and early detection of organ damage in SLE," Nature Reviews. Rheumatology, 2012.

[8] A. Doria, L. Iaccarino, A. Ghirardello et al., "Long-term prognosis and causes of death in systemic lupus erythematosus," The American Journal of Medicine, vol. 119, no. 8, pp. 700-706, 2006.

[9] A. Ippolito and M. Petri, "An update on mortality in systemic lupus erythematosus," Clinical and Experimental Rheumatology, vol. 26, pp. S72-S79, 2008.

[10] G. Bertsias and D. T. Boumpas, "Update on the management of lupus nephritis: let the treatment fit the patient," Nature Clinical Practice Rheumatology, vol. 4, no. 9, pp. 464-472, 2008.

[11] A. N. Kiani and M. Petri, "Quality-of-life measurements versus disease activity in systemic lupus erythematosus," Current Rheumatology Reports, vol. 12, no. 4, pp. 250-258, 2010.

[12] Y. N. Liao, C. S. Liu, T. R. Tsai et al., "Preliminary study of a traditional Chinese medicine formula in systemic lupus erythematosus patients to taper steroid dose and prevent disease flareup," The Kaohsiung Journal of Medical Sciences, vol. 27, no. 7, pp. 251-257, 2011.

[13] B. Y. Zhang, J. H. Dong, and Z. Y. Zhou, Traditional Chinese Internal Medicine, Shang Hai Science and Technology Press, Shanghai, China, 1985.

[14] D. P. Chen, C. K. Wong, P. C. Leung et al., "Anti-inflammatory activities of Chinese herbal medicine sinomenine and Liang Miao San on tumor necrosis factor- $\alpha$-activated human fibroblastlike synoviocytes in rheumatoid arthritis," Journal of Ethnopharmacologyvol, vol. 137, no. 1, pp. 457-468, 2011.

[15] Chinese Pharmacopoeia Commission, Pharmacopoeia of the People's Republic of China, People's Medical Publishing House, Beijing, China, 2005.

[16] X. F. Xu, H. R. Qu, Z. Y. Xiang et al., "Effects of Ziyin Jiedu Quyu Decoction on glucocorticoid dosage in systemic lupus erythematosus: a double-blind randomized controlled trial," Shang Hai Journal of Traditional Chinese Medicine, vol. 46, no. 1, pp. 33-36, 2012.

[17] H. H. Li and X. L. Lu, "An overview of Chinese Medicine in the treatment of systemic lupus erythematosus," Modern Journal of Integrated Traditional Chinese and Western Medicineno, vol. 21, no. 36, pp. 4100-4102, 2012.

[18] P. J. Zhao, J. C. Mao, X. J. Chen et al., "Efficacy observation on mild to moderate active systemic lupus erythematosus treated with compound Zishenqing formula," World Journal of Integrated Traditional and Western Medicine, vol. 7, no. 1, pp. 3739, 2012.

[19] X. J. Chen and J. H. Gu, "Observational study of Zi Shen Qing in the treatment of active systemic lupus erythematosus," Shang Hai Journal of Traditional Chinese Medicine, no. 10, pp. 16-18, 1999.

[20] X. J. Chen, J. H. Gu, J. C. Mao et al., "Clinical study of Zi Shen Qing combination with Western medicine in the treatment of systemic lupus erythematosus," Jiang Su Journal of Traditional Chinese Medicine, vol. 41, no. 8, pp. 26-28, 2009.

[21] S. Q. Liu and X. J. Chen, "Treatment of 62 mild to moderate active systemic lupus erythematosus with Zi Shen Qing formula," Hei Long Jiang Medicine Journal, vol. 123, no. 12, pp. 224226, 2010.

[22] W. J. Koopman, Arthritis and Allied Conditionsed, Williams \& Wilkins, Baltimore, Md, USA, 13th edition, 1997.

[23] The State Administration of traditional Chinese Medicine of the People's Republic of China, Guidance For Clinical Research of Chinese Herbal Medicine 2002, China Press of Traditional Chinese Medicine, Beijing, China, 2002.

[24] The State Administration of traditional Chinese Medicine of the People's Republic of China, Criteria of Diagnosis and Therapeutic Effect of Diseases and Syndromes in Traditional Chinese Medicine, Nanjing University Press, Beijing, China, 1994.

[25] X. Zhou, R. F. Xie, J. H. Gu et al., "Use HPLC-MS-MS to determine the astragaloside content of ZSQ granules," Shan Xi Journal of Traditional Chinese Medicine, vol. 29, no. 4, pp. 490492, 2008.

[26] X. Zhou, J. H. Gu, R. F. Xie et al., “The qualitative identification and quantitative detection for total paeoniflorin in ZSQ granules," Journal of Hangzhou Teachers College, vol. 28, no. 4, pp. 265-269.

[27] Z. Touma, M. B. Urowitz, S. Taghavi-Zadeh, D. Ibañez, and D. D. Gladman, "Systemic lupus erythematosus disease activity Index 2000 responder index 50: sensitivity to response at 6 and 12 months," Rheumatology, vol. 51, no. 10, pp. 1814-1819, 2012.

[28] C. S. Yee, V. T. Farewell, D. A. Isenberg et al., "The use of systemic lupus erythematosus disease activity index-2000 to define active disease and minimal clinically meaningful change based on data from a large cohort of systemic lupus erythematosus patients," Rheumatology, vol. 50, no. 5, Article ID keq376, pp. 982-988, 2011.

[29] D. Ibañez, D. D. Gladman, Z. Touma, M. Nikpour, and M. B. Urowitz, "Optimal frequency of visits for patients with systemic lupus erythematosus to measure disease activity over time," The Journal of Rheumatology, vol. 38, no. 1, pp. 60-63, 2011.

[30] P. P. Smith and C. Gordon, "Systemic lupus erythematosus: clinical presentations," Autoimmunity Review, vol. 10, no. 1, pp. 43-45, 2010.

[31] C. E. Tseng, J. P. Buyon, M. Kim et al., “The effect of moderatedose corticosteroids in preventing severe flares in patients with serologically active, but clinically stable, systemic lupus erythematosus: findings of a prospective, randomized, doubleblind, placebo-controlled trial," Arthritis \& Rheumatism, vol. 54, no. 11, pp. 3623-3632, 2006.

[32] J. M. Esdaile, "The efficacy of antimalarials in systemic lupus erythromatosus," Lupus, vol. 2, no. 1, pp. S3-S8, 1993.

[33] S. Q. Liu and X. J. Chen, “The effectiveness of Zi Shen Qing formula on regulation of CD4, CD25 with the mild to moderate active systemic lupus erythematosus patients," Liao Ning Journal of Traditional Chinese Medicine, vol. 34, no. 12, pp. 1669-1770.

[34] E. Whitley and J. Ball, "Statistics review 2: samples and populations," Critical Care, vol. 6, no. 2, pp. 143-148, 2002.

[35] H. Bootsma, P. Spronk, R. Derksen et al., "Prevention of relapses in systemic lupus erythematosus," The Lancet, vol. 345, no. 8965, pp. 1595-1599, 1995.

[36] M. F. Marmor, R. E. Carr, M. Easterbrook, A. A. Farjo, and W. F. Mieler, "Recommendations on screening for chloroquine 
and hydroxychloroquine retinopathy: a report by the american academy of ophthalmology," Ophthalmology, vol. 109, no. 7, pp. 1377-1382, 2002.

[37] T. Dörner, "Therapy: hydroxychloroquine in SLE: old drug, new perspectives," Nature Reviews Rheumatology, vol. 6, pp. 10-11, 2010.

[38] G. Ruiz-Irastorza, M. Ramos-Casals, P. Brito-Zeron, and M. A. Khamashta, "Clinical efficacy and side effects of antimalarials in systemic lupus erythematosus: a systematic review," Annals of the Rheumatic Diseases, vol. 69, no. 1, pp. 20-28, 2010.

[39] S. C. Chiu, Y. L. Lai, H. H. Chang et al., "The therapeutic effect of modified Yu Ping Feng San on idiopathic sweating in end-stage cancer patients during hospice care," Phytotherapy Research, vol. 23, no. 3, pp. 363-366, 2009.

[40] P. M. K. Poon, C. K. Wong, K. P. Fung et al., "Immunomodulatory effects of a traditional Chinese medicine with potential antiviral activity: a self-control study," The American Journal of Chinese Medicine, vol. 34, no. 1, pp. 13-21, 2006.

[41] J. H. Gu and X. J. Chen, "Animal experimental study on immune regulating function of ZSQ formula," Acta Universitatis Traditionis Medicalis Sinensis Pharmacologiaeque Shanghaino, vol. 15, no. 2, pp. 44-45, 2001.

[42] I. K. Zarkadis, D. Mastellos, and J. D. Lambris, "Phylogenetic aspects of the complement system," Developmental and Comparative Immunology, vol. 25, no. 8-9, pp. 745-762, 2001.

[43] M. M. Frank, J. P. Atkinson et al., Samter's Immunologic Diseases, Lippincott Williams \& Wilkins, Philadelphia, Pa, USA, 6 edition, 2001.

[44] Y. P. Chuang, C. H. Wang, N. C. Wang, D. M. Chang, and H. K. Sytwu, "Modulatory function of invariant natural killer T cells in systemic lupus erythematosus," Clinical and Developmental Immunology, vol. 2012, Article ID 478429, 8 pages, 2012.

[45] J. H. Gu and X. J. Chen, "Observation on compound Zishenqing on peripheral blood mononuclear Cell (PBMC) of active SLE by serum pharmacology," Shan Dong Journal of Traditional Chinese Medicine, vol. 25, no. 1, pp. 47-51, 2007.

[46] Z. Z. Yang, D. M. Grote, S. C. Ziesmer et al., "Soluble IL$2 \mathrm{R} \alpha$ facilitates IL-2-mediated immune responses and predicts reduced survival in follicular B-cell non-Hodgkin lymphoma," Blood, vol. 118, no. 10, pp. 2809-2820, 2011.

[47] E. M. El-Shafey, G. F. El-Nagar, A. S. El-Bendary, A. A. Sabry, and A. G. Selim, "Serum soluble interleukin-2 receptor alpha in systemic lupus erythematosus," Iranian Journal of Kidney Diseases, vol. 2, no. 2, pp. 80-85, 2008.

[48] R. K. Luijten, R. D. Fritsch-Stork, J. W. Bijlsma, and R. H. Derksen, "The use of glucocorticoids in Systemic Lupus Erythematosus. After 60 years still more an art than science," Autoimmunity Review, vol. 12, no. 5, pp. 617-628, 2012.

[49] G. Franchin and B. Diamond, "Pulse steroids: how much is enough?” Autoimmunity Reviews, vol. 5, no. 2, pp. 111-113, 2006.

[50] G. G. Illei, H. A. Austin, M. Crane et al., "Combination therapy with pulse cyclophosphamide plus pulse methylprednisolone improves long-term renal outcome without adding toxicity in patients with lupus nephritis," Annals of Internal Medicine, vol. 135, no. 4, pp. 248-257, 2001.

[51] M. Petri, "Monitoring systemic lupus erythematosus in standard clinical care," Best Practice and Research, vol. 21, no. 4, pp. 687-697, 2007.

[52] M. Thamer, M. A. Hernán, Y. Zhang, D. Cotter, and M. Petri, "Prednisone, lupus activity, and permanent organ damage," The Journal of Rheumatology, vol. 36, no. 3, pp. 560-564, 2009. 


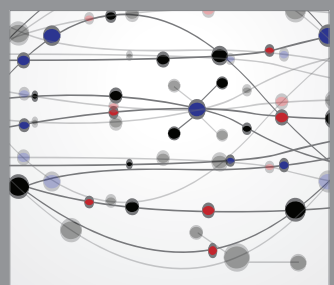

The Scientific World Journal
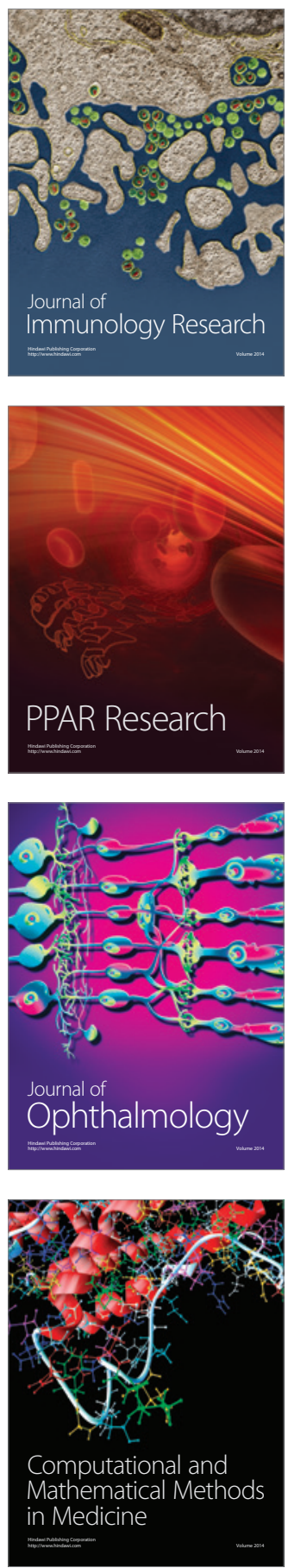

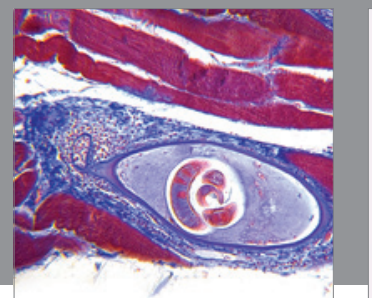

Gastroenterology

Research and Practice
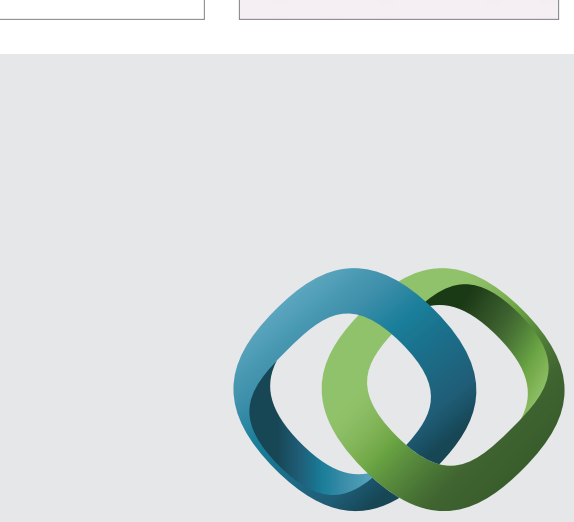

\section{Hindawi}

Submit your manuscripts at

http://www.hindawi.com
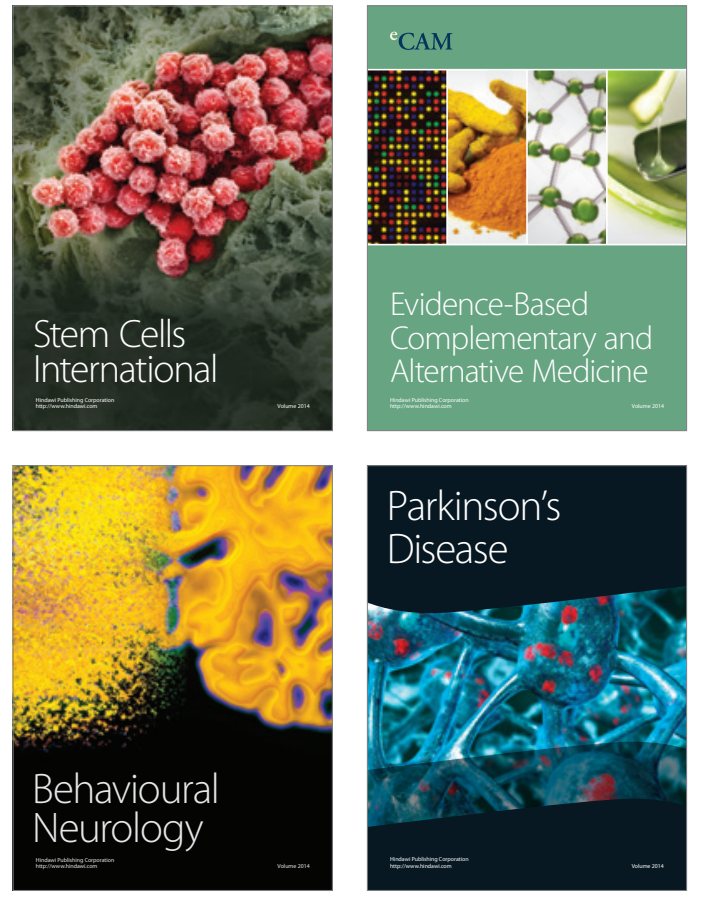
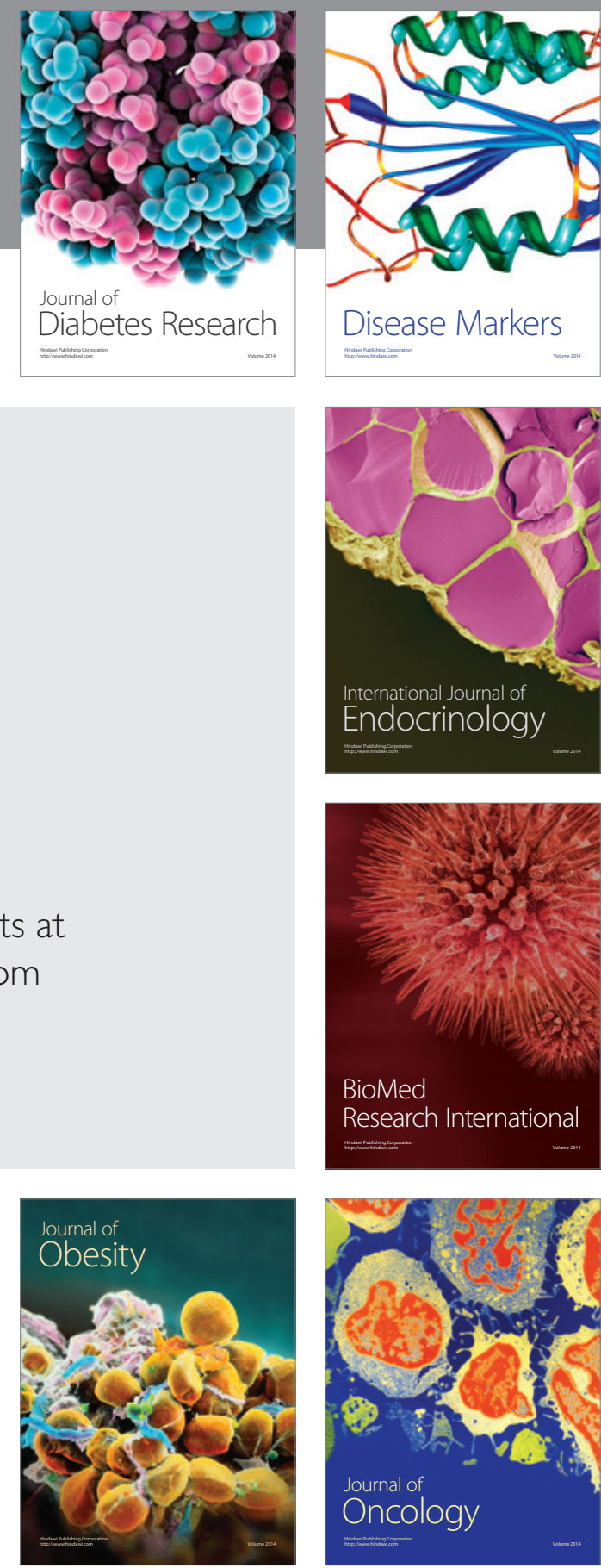

Disease Markers
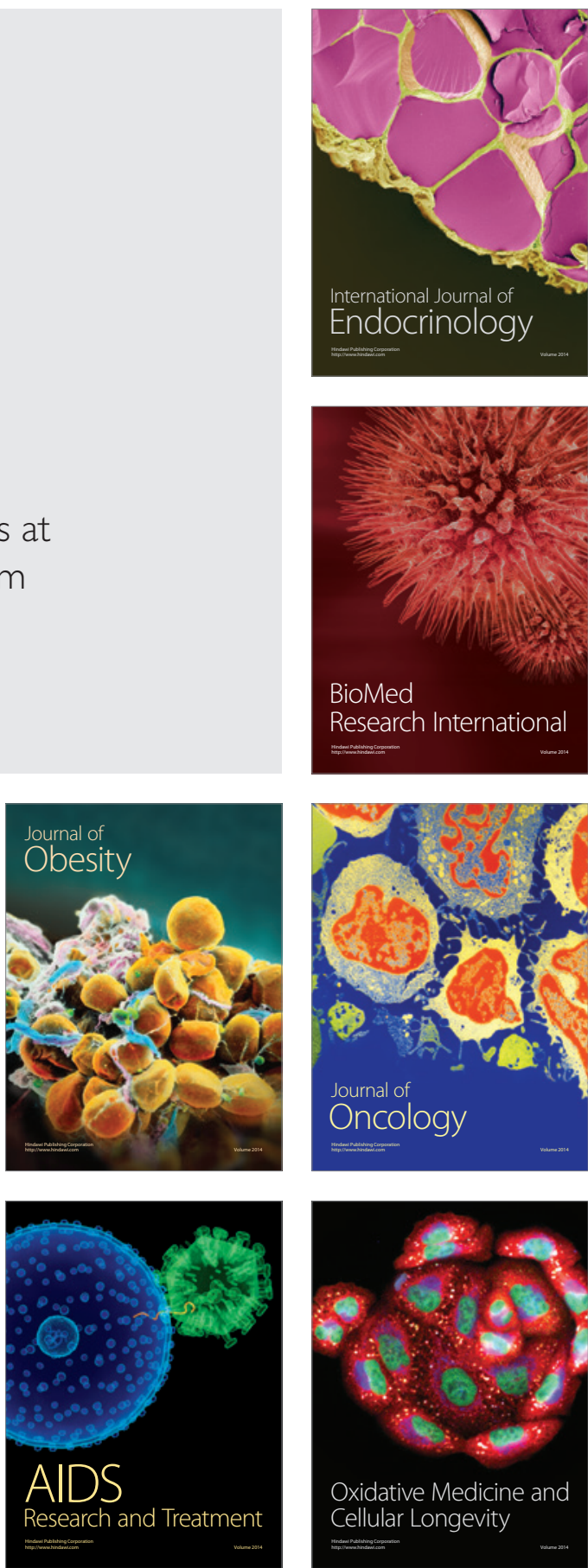